\title{
Experimental Evidence for Non-Thermal Contributions to Plasmon-Enhanced Electrochemical Oxidation Reactions
}

\author{
Marina Rodio*1,2 ${ }^{*}$, Matthias Graf ${ }^{* 3,4} \uparrow$, Florian Schulz ${ }^{2}$, Niclas S. Mueller ${ }^{5}$, \\ Manfred Eich $^{4,3}$ and Holger Lange ${ }^{1,2}$ \\ ${ }^{1}$ Hamburg Centre for Advanced Imaging of Matter, Luruper Chaussee 149, \\ Hamburg, 22761, Germany. \\ ${ }^{2}$ Institute of Physical Chemistry, University of Hamburg, Martin-Luther-King Platz 6, \\ Hamburg, 20146, Germany. \\ ${ }^{3}$ Institute of Materials Research, Helmholtz-Zentrum Geesthacht, Max-Planck-Strasse \\ 1, Geesthacht, D-21502, Germany \\ ${ }^{4}$ Institute of Optical and Electronic Materials, Hamburg University of Technology, \\ Hamburg, Eissendorfer Strasse 38, Hamburg, D-21073, Germany. \\ ${ }^{5}$ Department of Physics, Freie Universität Berlin, Berlin, Germany \\ $\dagger$ these authors contributed equally to this work \\ E-mail: marina.rodio@chemie.uni-hamburg.de \\ matthias.graf@tuhh.de
}

\begin{abstract}
Photocatalysis based on plasmonic nanoparticles has emerged as a promising approach to facilitate light-driven reactions under far milder conditions than thermal catalysis. Several effects, such as strong local electromagnetic fields, increased electron and lattice temperatures, or the transfer of non-thermal charge carriers could contribute to the reaction rate enhancement. In order to understand plasmon-enhanced catalysis and to enable plasmonic platforms, a distinction between the different underlying effects is required. We investigate the electrochemical model reactions oxidative hydroxide adsorption and glucose oxidation and deconvolve the enhancement processes via their dependence on excitation wavelength. We observe that non-thermal effects contribute significantly to the plasmonic enhancement.
\end{abstract}

\section{Introduction}

Plasmonic nanoparticles (NPs) are currently intensively discussed as an enhancing agent for light-driven heterogeneous catalytic conversions. ${ }^{1-6}$ The possibility of photo-generated carriers initiating the reaction might lower its operating temperature, which is usually a problem in terms of catalyst stability, high required thermal input and reaction selectivity. Thus, an operating temperature reduction is desirable. ${ }^{7}$ In a pioneering work, Christopher et al. observed a strong increase of the catalytic reaction rate on plasmonic silver nanoparticles upon illumination with visible light and a scaling of the rate enhancement with the incident optical power. ${ }^{8}$ The authors proposed a combination of electromagnetic field enhancement and extracted electrons to be responsible for the enhancement. However, their early model did not account for the currently discussed specifics of the plasmon excitation. ${ }^{4,9-14}$ Looking at gold nanoparticles (AuNPs) as plasmonic model system, different processes could contribute to the reaction rate enhancement (cf. Figure 1):

1) During the coherent drive of the plasmon, light is focused into strongly enhanced, localized electric fields around the NPs. ${ }^{15}$ These strong fields could affect the energies of molecular (anti-)bonding states e.g. of adsorbates involved in the reaction, as for example suggested for the dissociation of $\mathrm{O}_{2}{ }^{16}$

With the subsequent plasmon decay, several processes occur that could also lead to reaction rate enhancements: $:^{4,9,17-20}$

2) Landau damping of the plasmon results in a non-equilibrium, non-thermal carrier distribution, i.e. electrons and holes are excited to states far from the Fermi level. These could enhance the reaction via a direct ${ }^{21}$ or indirect ${ }^{22-26}$ carrier transfer between the NPs and the adsorbate-binding molecular orbitals. ${ }^{2,4,25,27,28}$ 
3) Carrier-carrier scattering leads to an equalization of the non-thermal carrier distribution, resulting in a bath of thermalized excited electrons in equilibrium. Within 10-1000 fs these could also populate electronic states in the adsorbate molecule. ${ }^{18}$

4) Electron-phonon coupling occurs on picosecond timescales and turns the thermalized carrier bath into local heat (phonons). ${ }^{19,20}$ The elevated NP temperatures could enhance a phonon-driven chemical reaction that occurs at or close to the surface.

reaction-enhancing process

enhanced near-fields

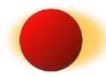

non-thermalized hot carriers

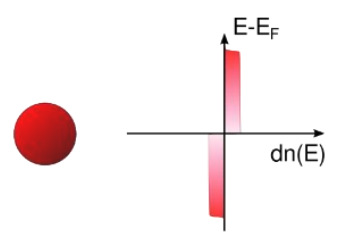

thermalized hot carriers
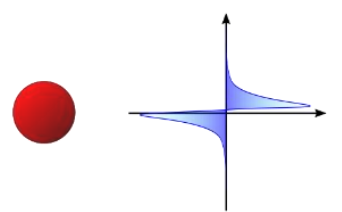

local heating

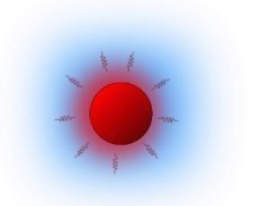

$\lambda$-dependent reaction

rate enhancement

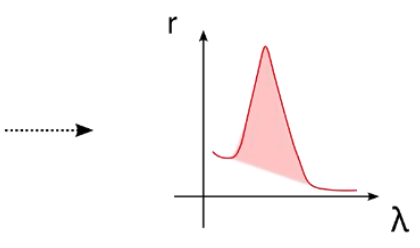

$\lambda$
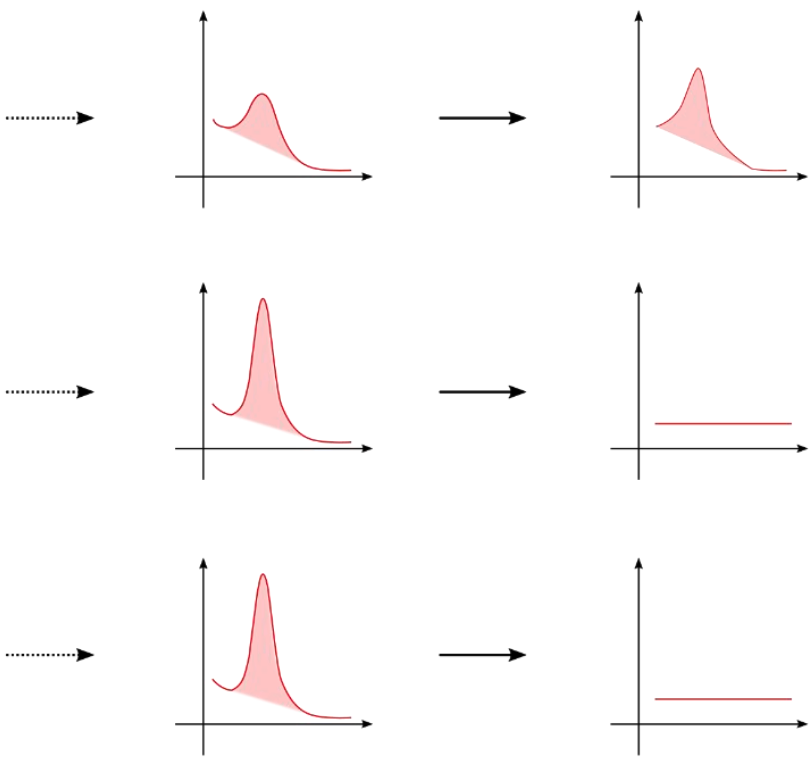

$\lambda$-dependent, normalized

reaction rate enhancement

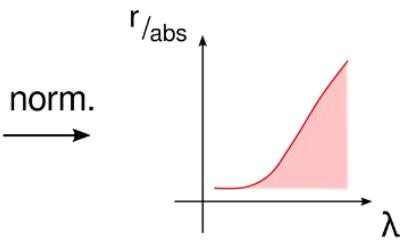


below can only generate a thermal enhancement) with the direct (no barrier layer so that all enhancement mechanisms can occur on the metal side) excitation of the plasmon pointed towards significant nonthermal contributions. ${ }^{37}$

The possible contributing processes have different dependencies on the excitation conditions (cf. Figure $1)$ :

\section{Enhanced near fields}

The local fields around AuNPs are strongly enhanced when exciting in resonance with the plasmon. ${ }^{15}$ The excitation-wavelength dependence of the near field enhancement does in general not track the absorption, which is known as near-field far-field shift. ${ }^{38}$ In the case of small AuNPs, interband transitions lead to a decrease in local field enhancement at short wavelengths and an increase in absorption. ${ }^{15,39}$ On the other hand, the absorption drops faster towards the near-infrared than the field enhancement, because it scales linearly with the excitation energy (apart from the plasmon resonance), while the field enhancement does not. ${ }^{15}$ Figure 2 displays a model calculation for small AuNPs. The field enhancement normalized to the absorption possesses an excitation-wavelength dependence. In case the population of the reactants' molecular (anti-)bonding orbitals promoted by the local field would be the main contribution to the reaction rate enhancement, the normalization to the absorbed optical power would lead to no clear resonance, but to a rate increase towards longer wavelengths (Figure 2, inset). The complicated dependencies of the electronic structure of adsorbed molecules makes the scaling of the reaction rate enhancement with incident power hard to define. However, several studies report surface-enhanced Raman scattering as field driven process to scale super linear with power. ${ }^{40-42}$

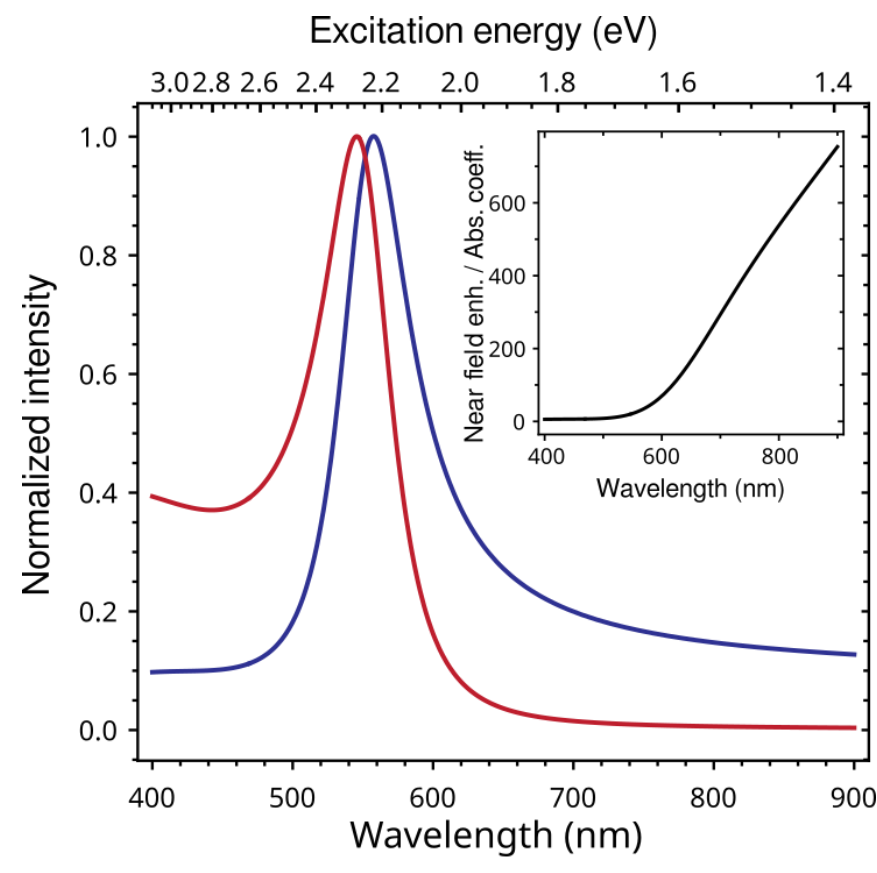

- Average field enh. - Absorption coeff.

Figure 2: Calculated absorption cross section and field enhancement, averaged over the surface of a 12 $\mathrm{nm}$ AuNP using Mie theory. The refractive index of the surrounding medium was set to $\mathrm{n}=1.6$ to account for the ITO substrate and the molecules surrounding the gold nanoparticle. An analytic model from Ref. ${ }^{43}$ was used for the dielectric function of gold. Inset: Average near field intensity enhancement, normalized to the calculated absorption cross section. 
Landau damping is a momentum-conserving process that allows donating the full photon energy into a single intraband transition, resulting in non-thermal electrons and holes. ${ }^{12}$ In this case, the exact carrier distribution depends on the excitation wavelength. ${ }^{14,44}$ Absorbed low-energy photons result in electrons excited close to the Fermi edge. Photons with higher energies can excite non-thermal electrons far from the Fermi edge. ${ }^{14,26,45}$ Even higher photon energies can excite interband transitions from the d-band into states above the Fermi level. ${ }^{13}$ For the case that non-thermalized hot electrons are the main contribution to the reaction rate enhancement, a spectrum of the reaction rate enhancement normalized to the absorbed number of photons would show a peak in the spectral range where hot electrons are generated most effectively.

\section{Thermalized hot carriers}

The lifetimes of non-equilibrium carriers is very short (100 fs) and their excess energy is quickly redistributed within the electron gas. ${ }^{12,13}$ As the estimated heat capacity of the electron gas in AuNPs is a linear quantity in the temperature range up to $3000 \mathrm{~K}$ and independent of the excitation-mechanism, ${ }^{11}$ the temperature of the thermalized electron gas only depends on the amount of absorbed optical energy. Consequently, for thermalized hot carriers as the main contribution to the reaction rate enhancement, a normalization to the absorption would result in a vanishing wavelength dependence.

Both, non-thermalized and thermalized hot carrier-based enhancements would lead to a linear increase of the reaction rate with absorbed optical power due to the linearity between photon absorption and carrier generation.

\section{Local heating}

The heat capacity of AuNPs does not differ significantly from bulk Au. The rise in temperature thus depends linearly on the total amount of absorbed optical energy. ${ }^{10,11,46}$ If temperature was the main reaction rate enhancing contribution, the wavelength dependence of the rate enhancement would reproduce the absorption spectrum. Similar to the case of thermalized hot carriers, the reaction rate enhancement, normalized to the absorbed optical power would not show any clear dependence on the excitation.

When assuming the rate of a chemical reaction scaling in an Arrhenius type with the temperature, the reaction rate would increase exponentially with increasing incident optical power for sole thermally enhanced processes.

To quantify the excitation-wavelength dependence experimentally, a reaction with a directly observable rate is required. When regarding the charge flow between the plasmonic metal and its surrounding as an electrical current that occurs due to illumination under defined conditions, one can directly relate this current to the number of carriers contributing to the reaction's enhancement. This suggests an electrochemical approach in which the plasmonic metal represents the working electrode under investigation. The charges extracted from/into the adjacent phase (electrolyte) are compensated at a counter electrode delivering an externally measurable current. This approach enables to screen an arbitrary reaction (cathodic, i.e. extraction of electrons from the plasmonic metal working electrode, or anodic, the extraction from or compensation of holes in the metal) by applying the respective necessary electrochemical potential between this working electrode and an inert reference electrode (i.e. at which no charge transfer reactions occur). In this setup we used AuNPs immobilized on an optically passive and electrochemically inert electrode (In-doped $\mathrm{SnO}_{2}$, ITO) to compare the reaction current under dark with that under defined illumination conditions. The difference of both over time is averaged giving the photocurrent $I_{\mathrm{ph}}$, a quantitative measure of the steady-state photo-enhancement of the reaction rate. As model reactions with broad relevance we screened the alkaline adsorption of hydroxide on the $\mathrm{Au}$ surface and - upon addition of glucose to the electrolyte - the oxidation of glucose. ${ }^{23,47-50} \mathrm{We}$ varied power and wavelength of the optical excitation to disentangle thermal and non-thermal influences. 
We observed linear dependencies of the reaction rates on the incident optical power, whereas its effectivity (slope of $I_{\mathrm{ph}}$ vs. absorbed power) strongly varies with the excitation wavelength, showing a maximum at the plasmon resonance. The normalization to the absorbed optical power allows us to conclude that the main contribution to the reaction rate enhancement are non-thermal carriers and not thermalized electrons, elevated NP temperatures or strong local fields.

\section{Results \& Discussion}

AuNPs were synthesized with a mean diameter of $12.3 \pm 0.8 \mathrm{~nm}$ (see Supporting Information Figure S1) and immobilized on an ITO-covered glass slide to prepare AuNP/ITO electrodes. As apparent in the scanning electron microscopy (SEM) images (Figures 3a-b), AuNPs cover the ITO surface with negligible agglomerate formation. SEM investigations (see Supporting Information Figure S2) reveal no relevant AuNP detachment from the substrate after the electrochemical experiments.
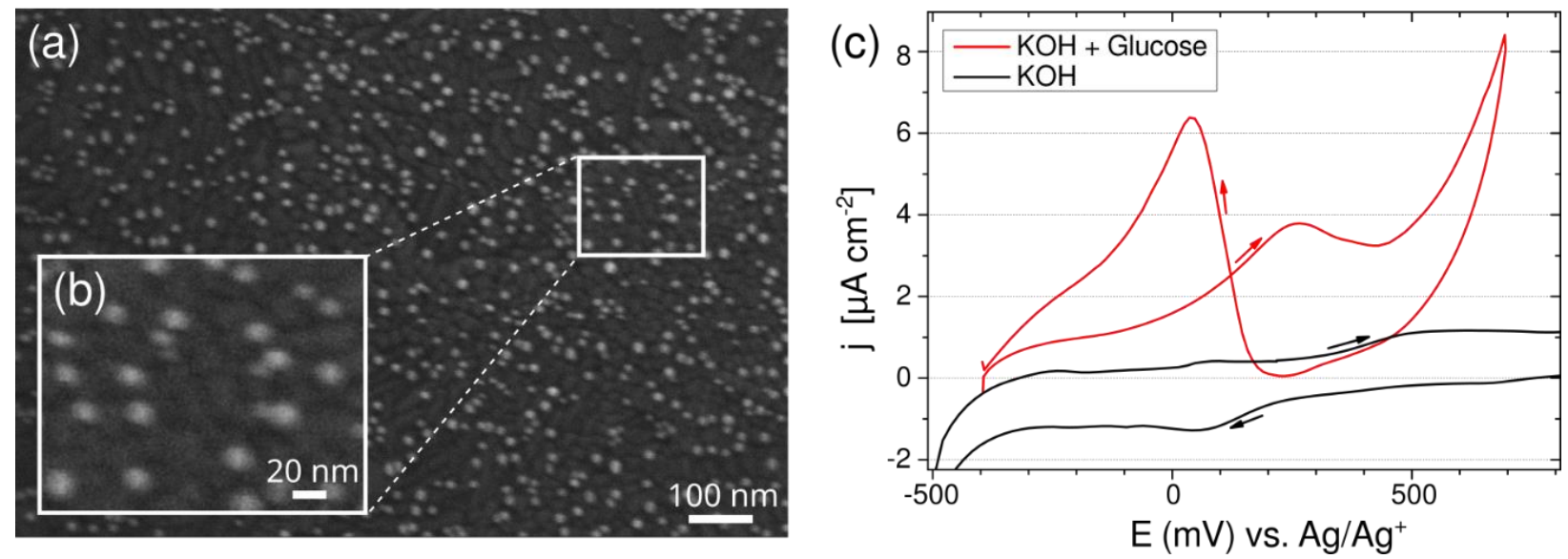

Figure 3: (a,b) SEM images of the AuNP-ITO electrode surface. (c) Cyclic voltammogram in glucosecontaining $(500 \mathrm{mM})$ and glucose-free base electrolyte $(500 \mathrm{mM} \mathrm{KOH})$. The current was normalized to the AuNP electrochemically active surface area (ECSA, see Supporting Information Figure S3) to give the current density j. Scan rate: $50 \mathrm{mV} / \mathrm{s}$. Arrows indicate the scan directions.

The electrodes were used for the glucose oxidation in alkaline environment $(\mathrm{pH}=14)$.

In alkaline environment, alcohols (as which we consider glucose) deprotonate to alkoxides which are then oxidized on a surface decorated with oxide species. ${ }^{47,51,52}$ The latter result from hydroxide adsorption according to:

$$
\mathrm{Au}+\mathrm{OH}_{\mathrm{aq}}^{-} \rightarrow \mathrm{Au}-\mathrm{OH}_{\mathrm{ads}}+\mathrm{e}^{-}
$$

which occurs at potentials similar to the oxidation potential of glucose. Glucose oxidation to gluconic acid then occurs via a one-electron transfer between the metal and the electrolyte ${ }^{48}$ :

$$
\mathrm{C}_{6} \mathrm{H}_{12} \mathrm{O}_{6}+\mathrm{Au}-\mathrm{OH}_{\mathrm{ads}} \rightarrow \mathrm{Au}-\mathrm{C}_{6} \mathrm{H}_{12} \mathrm{O}_{7, \text { ads }}+\mathrm{H}^{+}+\mathrm{e}^{-}
$$

Recently, Wang et al. demonstrated the photo-enhancement of alkaline glucose oxidation on AuNPs. ${ }^{23}$ A broad range of parameters that affect the photo enhancement of the reaction were reported, i.e. the incident light wavelength, the power of the light source and the $\mathrm{pH}$ of the electrolyte solution.

The current between the AuNP/ITO electrode and the counter electrode serves as a metric to quantify the extent of potential and illumination-dependent charge transfer. The required potential range for glucose oxidation was identified by cyclic voltammetry (CV) measurements under dark conditions (Figure 3c). The voltammogram (red curve) shows a broad oxidation peak at approximately $+300 \mathrm{mV}$ upon anodic potential $(E)$ increase, which is attributed to the oxidation of glucose to gluconic acid 
according to Eq. (2) ${ }^{49,50}$ Upon further potential increase, the peak slowly decays due to a saturation of the surface with adsorbing gluconic acid species $\left(\mathrm{Au}-\mathrm{C}_{6} \mathrm{H}_{12} \mathrm{O}_{7, \text { ads }}\right)$ and further increases at potential ranges where higher oxides form ${ }^{53}$ according to:

$$
\mathrm{Au}-\mathrm{OH}_{\mathrm{ads}}+\mathrm{OH}_{\mathrm{aq}}^{-} \rightarrow \mathrm{Au}-\mathrm{O}_{\mathrm{ads}}+\mathrm{H}_{2} \mathrm{O}+\mathrm{e}^{-}
$$

and the oxidation of the initially non-covered Au sites:

$$
2 \mathrm{Au}+6 \mathrm{OH}_{\mathrm{aq}}^{-} \rightarrow \mathrm{Au}_{2} \mathrm{O}_{3}+3 \mathrm{H}_{2} \mathrm{O}+6 \mathrm{e}^{-}
$$

In the cathodic scan, i.e. after scan reversal, a second oxidation peak at approximately $+100 \mathrm{mV}$ is observed, which can also be attributed to the adsorption-controlled glucose oxidation onset in coincidence with the onset of stripping of gluconic acid and/or oxygen species from the oxide-covered $\mathrm{Au}$ (at $+200 \mathrm{mV}$, according to the reverse of Eq.s (1), (3) and (4)). ${ }^{47,54}$ The observed behavior is equal to that observed earlier for AuNP/ITO electrodes..$^{48}$ This hydroxide adsorption was screened in a separate experiment using a glucose-free electrolyte. The corresponding curve in Figure $3 \mathrm{c}$ shows the hydroxide adsorption onset during the anodic scan at approximately $0 \mathrm{mV}$ and occurring throughout the entire anodic potential range, originating from the oxide formation-stripping behavior of $\mathrm{Au}$ in alkaline conditions. ${ }^{53}$ The subsequent scan reversal leads to a slight oxide stripping peak at $+50 \mathrm{mV}$ followed by the reduction of dissolved oxygen residuals.

To assess the plasmon-based reaction enhancement, we carried out chronoamperometry measurements (i.e. a potential step from the open-circuit potential to a potential in the glucose oxidation and simultaneous Au surface oxidation regime as identified by CV under dark conditions; see Supporting Information Figures S4A-B for the corresponding current transients under dark and illumination).

All transients show a current decrease in the first seconds of the experiments, which we attribute to the transport of electroactive species to the electrode and capacitive charging upon switch-on. At later times of the experiment (after $30 \mathrm{~s}$ ) the currents were stable. To obtain the illumination-related current transient (photocurrent $I_{p h}(t)$ ), we subtracted the dark current from that of the illuminated electrode. $I_{\mathrm{ph}}(t)$ was averaged within the time interval $[30 ; 60 \mathrm{~s}]$ to obtain the steady state photocurrent $I_{\mathrm{ph}}$ (cf. Supporting Information Figures S4C-D). Figure 4 displays $I_{\mathrm{ph}}$ for different applied potentials $E$ and in different electrolyte solutions. We observe a small photocurrent over the entire $E$ range in the (glucosefree) base electrolyte whereas the photocurrent steadily increases when glucose is present. Relative to the dark current, the current enhancement in the base electrolyte is large at lower potentials, i.e. where the hydroxide monolayer adsorption sets on (due to the normalization to small dark currents, the relative enhancement is apparently high at potentials before this onset although photocurrents are in fact low) and decreases with increasing potential. In contrast, the current enhancement has a clear maximum at $E=+300 \mathrm{mV}$ when glucose is present (coinciding with the $\mathrm{CV}$ peak potential, Figure 3c). We conclude from this similarity of the $\mathrm{CV}$ peak with the relative $I_{\mathrm{ph}}$ peak that the photo-enhancement of the glucose oxidation rate is proportional to its dark oxidation rate. ${ }^{23}$ Although an electrode potential shift of a few $\mathrm{mV}$ due to illumination has been reported, ${ }^{55}$ the effect is not expected to lead to the observed large current increase and is furthermore immediately compensated by the potentiostat. Therefore, it can be neglected. We thus focused on $+300 \mathrm{mV}$ as the working potential. We assume that in case of the glucose-containing electrolyte at this potential both reactions (hydroxide adsorption by Eq. (1) and glucose oxidation according to Eq. (2)) occur whereas only the first proceeds in the glucosefree electrolyte. 


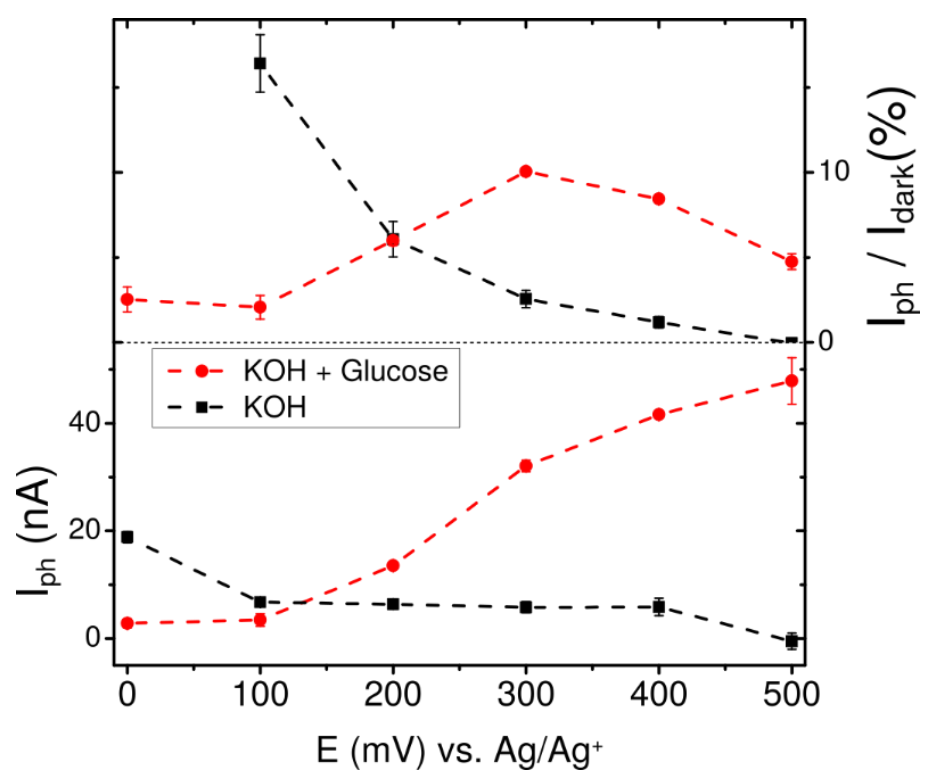

Figure 4: Photoresponse of AuNP/ITO electrodes glucose oxidation and hydroxide adsorption. Absolute (left ordinate, dashed lines) and relative (ratio of $I_{\mathrm{ph}}$ to dark current $I_{\mathrm{dark}}$, right ordinate, straight lines) photocurrents vs. applied potential $E$, excited at $454 \mathrm{~nm}$ with $35.7 \mathrm{~mW}$ in the glucosecontaining (red) and the glucose-free base electrolyte (black). Error bars represent the standard deviation within the steady-state duration of the experiment.

$I_{\mathrm{ph}}$ versus illumination wavelengths $\lambda$ from $454-802 \mathrm{~nm}$ is displayed in Figure 5a.
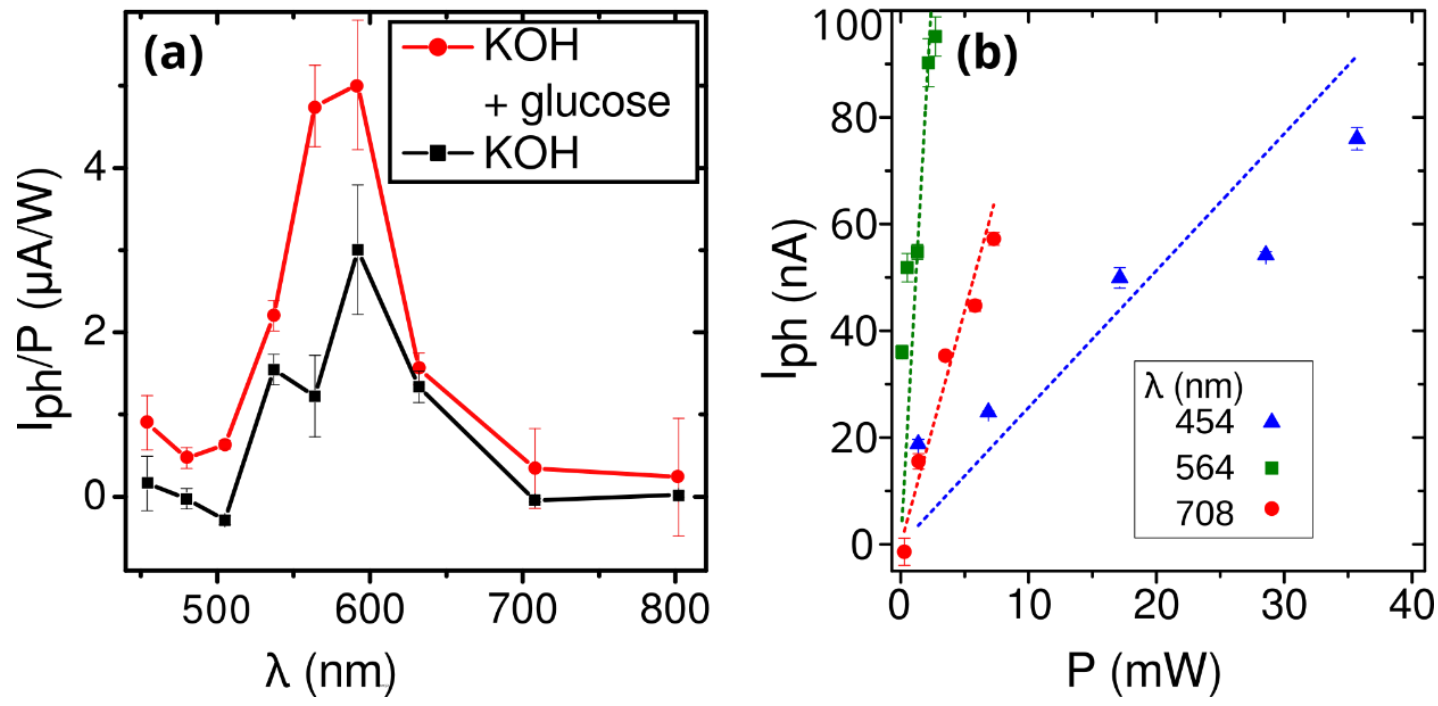

Figure 5: (a) Photocurrent $I_{\mathrm{ph}}$ vs. excitation wavelength $\lambda$ for both electrolytes at $E=+300 \mathrm{mV}$. The measured values are normalized to the incident optical power (cf. Supporting Information Figure S5 and Figure 1, central column). (b) $I_{\mathrm{ph}}$ vs. incident optical power $P$, at different excitation wavelengths.

The data correspond to the situation displayed in the central column of Figure 1. As expected from the preceding discussion, we observe a strong dependence of $I_{\mathrm{ph}}$ on $\lambda$ with a pronounced maximum around the plasmon resonance in the glucose oxidation case. $I_{\mathrm{ph}}$ for increasing incident optical power at different excitation wavelengths is displayed in Figure $5 \mathrm{~b} . I_{\mathrm{ph}}$ scales linearly with the power in all cases. These two observations alone would apply to all potential reaction rate-enhancing processes. 

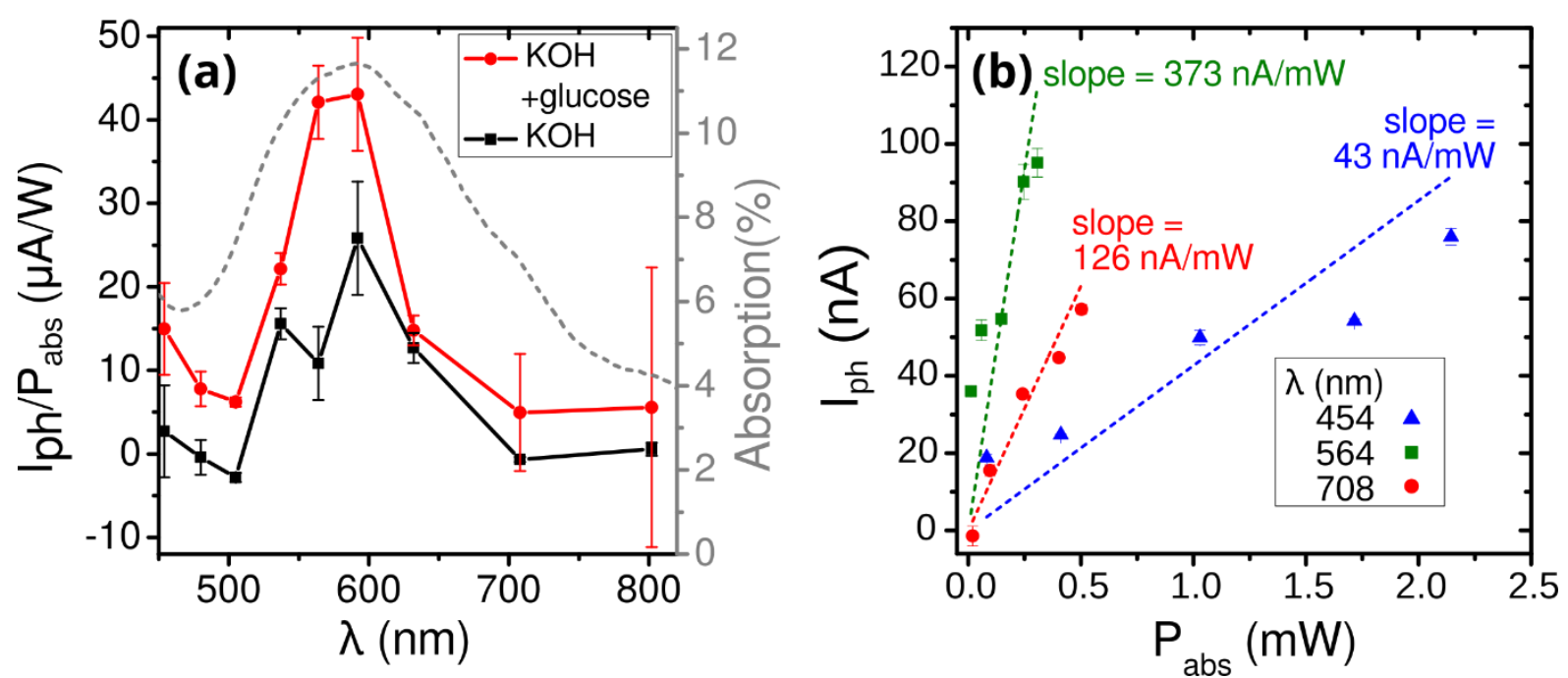

Figure 6: (a) Photocurrent $I_{\mathrm{ph}}$ normalized to the absorbed optical power $P_{\mathrm{abs}}$ vs. excitation wavelength $\lambda$ for both electrolytes at $E=+300 \mathrm{mV}$. The gray dashed line refers to the right ordinate and represents the optical absorption of the AuNP/ITO electrode. (b) $I_{\mathrm{ph}}$ vs. absorbed optical power $P_{\mathrm{abs}}=P \cdot A$ for different excitation wavelengths, including linear fits and the corresponding slopes at the same $E$ as in (a).

$I_{\mathrm{ph}}$ scales linearly with the reaction rate enhancement via the number of electrons excited via absorption. The normalization of $I_{\mathrm{ph}}$ to the optical absorption of the electrode then corresponds to the situations displayed in the right column of Figure 1, as discussed above.

To measure the absorption, micro-absorption spectroscopy was applied (see dashed line in Figure 6a). The experiment accounts for the reflected and transmitted light (see Supporting Information Figure S6). The spectrum of $I_{\mathrm{ph}}$ normalized with the absorbed power $\left(P_{\mathrm{abs}}=P \cdot\right.$ Absorption) (Figure 6a) clearly displays a non-vanishing dependence on the excitation wavelength, for both electrolytes showing a maximum around $\lambda=590 \mathrm{~nm}$. We find higher rate-enhancements in the glucose-containing solution than in the base electrolyte by a factor of 1.7. According to Eq. (2), glucose oxidation first demands for an adsorbed $\mathrm{OH}$ species (i.e. one charge to be transferred). The oxidation of glucose then demands for another charge. We can deduce that the enhancement refers to both reactions (1) and (2), while deconvolving both appears rather speculative due to different adsorbate coverages in both cases. The retaining wavelength dependence of the normalized $I_{\mathrm{ph}}$ (as a measure for the reaction rate enhancement) provides a strong indication that non-thermal effects contribute significantly to the enhancement of both oxidation reactions (apparently, the photoexcitation enhancement of the glucose oxidation occurs via a similar mechanism as the hydroxide adsorption, whereas the enhancement is stronger for glucose oxidation). As discussed earlier, thermal effects would vanish after normalization to the absorption and local fields would show a more linear dependence in the observed wavelength regime. $I_{\mathrm{ph}}$ is independent from the duration of illumination (see Supporting Information Figures S4C-D), i.e. from any heat uptake. The slope of the power dependence of $I_{\mathrm{ph}}$ (Figure 6b) normalized to the absorbed optical power $P_{\mathrm{abs}}$ represents the effectivity of the reaction rate-enhancing process. It is a measure for the reaction events per absorbed photon energy. Thermal processes would show a non-linear behavior with increasing power (see discussion above). This is observed in none of the regarded cases. For non-thermal carriers, the slope is expected to be linear and larger for wavelengths where non-thermal carriers are excited effectively. Following the present understanding of the plasmon decay, this is what we observe: Landau damping of the plasmon is effectively a plasmon-electron scattering process, where the energy of a 
single plasmon quantum generates an electron-hole pair. ${ }^{13}$ Plasmon excitations thus effectively generate non-thermal carriers, where the excess energy scales with the photon energy. ${ }^{22,56,57}$ Both of these two important and exemplary reactions seem to be enhanced by the injection of non-thermal carriers resulting from the plasmon decay, but each with different sensitivity. Therein, the plasmonic enhancement of hydroxide adsorption, being a prerequisite to many alkaline oxidation reactions, further emphasizes the potential of hot carrier injection for e.g. oxygen evolution or green fuel (e.g. methanol) formation reactions. In order to achieve an even more efficient enhancement by hot charge carriers, further studies addressing the generation of non-equilibrium carrier distributions, as well as their lifetimes and diffusion are important. ${ }^{12,58}$ Furthermore, the observed difference in reaction enhancement between the oxidation and the hydroxide adsorption points towards complex and convolved dependencies.

\section{Conclusions}

In this work, we investigated the plasmon-enhanced electrochemical glucose oxidation under hydroxide adsorption on AuNPs that are attached to an ITO electrode in an alkaline electrolyte. The photocurrent, i.e. the difference between illuminated and dark current in the steady state, is suitable to quantify a steady state reaction enhancement under minimization of capacitive effects. We estimated the wavelengthdependent photocurrent to separate possible reaction rate enhancing processes. When normalized to the absorbed power, the reaction rate enhancement shows a distinct wavelength dependence with a maximum coinciding with the plasmon resonance. This is not expected neither for thermal nor for processes supported by local fields. Thus, non-thermal carriers seem to be most relevant for the plasmonassisted conversion enhancement. 


\section{Acknowledgements.}

We thank Patryk Kusch (FU Berlin) for fruitful discussions on the scaling of local fields and Stephanie Reich (FU Berlin) for support with micro-absorption measurements.

This work was financially supported by the Cluster of Excellence 'Advanced Imaging of Matter' of the Deutsche Forschungsgemeinschaft (DFG) - EXC 2056 - project ID 390715994. M.R. is supported by the Louise Johnson Fellowship via the Cluster of Excellence "Advanced Imaging of Matter'. F.S. is supported by the DFG via the project SCHU 3019/2-1. M.R. and M.G. contributed equally to this work. N.S.M. was supported by the European Research Council (ERC) under grant DarkSERS (772108).

\section{Materials and Methods.}

Chemicals. Tetrachloroauric(III) acid ( $\geq 99.9 \%$ trace metal basis), trisodium citrate dihydrate $(\geq 99.0 \%)$, 11-mercaptoundecanoic acid (95\%) were purchased from Sigma-Aldrich; ethanol absolute (100.0\%) was obtained from VWR. Ethylenediaminetetraacetic acid tetrasodium salt hydrate (EDTA) was obtained from Merck. Glucose was purchased from Sigma and used as received. $\mathrm{KCl}$ was purchased from Nanjing Chemical Reagent Co., Ltd. (Nanjing, China). ITO-covered glass (surface resistivity 8-12 $\Omega / \mathrm{cm}^{2}$ was purchased from Sigma-Aldrich. All aqueous solutions were prepared with Millipore water (resistivity of $18.2 \mathrm{M} \Omega \cdot \mathrm{cm}$ ). $\mathrm{HClO}_{4}$ and $\mathrm{KOH}$ were purchased from Carl Roth, Germany.

AuNP synthesis. Citrate-stabilized AuNPs were synthesized and characterized as published earlier. ${ }^{59} \alpha$ Methoxypoly(ethylene glycol)- $\omega$-(11-mercaptoundecanoate) (PEGMUA, molecular mass: $2 \mathrm{kDa}$ ) was synthesized as described previously.$^{60}$ AuNPs were functionalized by straightforward ligand exchange of citrate-stabilized AuNPs using a mixture of PEGMUA and MUA in aqueous solution as reported: ${ }^{61}$ A PEGMUA/MUA mixture was prepared by mixing different volumes of an aqueous solution of PEGMUA ( $1 \mathrm{mM})$ and an ethanolic solution of MUA $(1 \mathrm{mM})$, yielding a mixture with the molar PEGMUA/MUA ratios of 1:3. A solution of PEGMUA/MUA-AuNPs with a final concentration of AuNP of $10 \mathrm{nM}$ was prepared.

AuNPs characterization. Transmission Electron Microscopy (TEM) measurements were performed using a Jeol JEM-1011 instrument operating at $100 \mathrm{kV}$. For TEM sample preparation, $10 \mu \mathrm{L}$ of sample solution was drop-casted onto a carbon-coated copper grid, which was placed on a glass slide, and left to dry for at least $24 \mathrm{~h}$. Quantitative analyses of AuNP size distributions based on TEM measurements were conducted using ImageJ.

AuNPs immobilization on ITO substrate. AuNPs were modified on an ITO slide for cyclic voltammetry measurements. Before modification, the ITO substrate with a surface area of $1.44 \mathrm{~cm}^{2}(1.2 \mathrm{~cm} \mathrm{x} 1.2 \mathrm{~cm})$ was cleaned under ultrasonic agitation subsequently in acetone, isopropanol and water for $15 \mathrm{~min}$ each. The slide was thoroughly dried with nitrogen gas, and treated with a PSD-UV4 $\mathrm{O}_{2}$ plasma cleaner system (Novascan 203 Technologies) for $30 \mathrm{~s}$ to further remove organic contaminants from the surface and make the glass hydrophilic. The cleaned slide was then immersed into a freshly prepared diluted acidic solution $(p H=2)$ of AuNPs for $72 \mathrm{~h}$. The obtained AuNPs/ITO electrode was rinsed with water to clean from non-immobilized NPs, and dried with nitrogen gas. The electrochemically active surface area of this electrode was determined via the capacitance ratio method with $6.69 \mathrm{~cm}^{2}$ (see Supporting Information Figure S3, this area corresponds to a geometrical electrode area of $1.44 \mathrm{~cm}^{2}$ ) and served to normalize the current observed $\mathrm{CV}$ experiments.

AuNPS/ITO electrode characterization. AuNPs/ITO characterization was performed by Scanning Electron Microscopy (SEM) using a Leo Gemini 1530 microscope.

Micro-absorption measurements. The optical setup for micro-absorption measurements was described recently. ${ }^{62}$ Briefly, a supercontinuum laser (Fianium, SC-400-4, 450-2400 nm) was used as a light source. The light was guided through a linear polarizer and a beam splitter (Thorlabs, BSW26R) into an inverted microscope (Olympus, IX71). A Leica HCX PL Fluotar 100× objective with a numerical aperture (NA) of 0.9 was used to focus the light onto the sample with a laser power $<100 \mu \mathrm{W}$. The AuNPs/ITO sample was mounted to a motorized xy-translation stage. The position of the laser spot on 
the sample was visible through a microscope camera. The transmitted light intensity $(T)$ was collected with an Olympus Mplan FL N BD 100× objective with an NA of 0.9 and guided by a fiber (Ocean Optics, QP600-2-UV-BX for 450-950 nm and BIF600-VIS-NIR for 700-1200 nm) to a spectrometer (Avantes, Avaspec 3648). The reflected light intensity $(R)$ was separated from the incoming light with a second beam splitter (Thorlabs, BSW26R) and detected by a spectrometer (same fibers and spectrometer as for the transmitted light). The absorbed light intensity $(A)$ was determined by subtracting $T$ and $R$ contributions from the source light intensity.

Electrochemical and photoelectrochemical measurements. Electrochemical and photoelectrochemical measurements were performed using a ZenniumX potentiostat and a photoelectrochemical cell by Zahner, Germany, equipped with a Pt wire counter electrode. AuNP/ITO electrodes were contacted with Au wire using Ag paste (Plano, Germany) and nail polish for electrical insulation. ECSA of all samples was determined using the capacitance ratio method $^{63}$ i.e. performing cyclic voltammetry $(\mathrm{CV})$ cycles under varied scan rates of 2,5 and $10 \mathrm{mVs}^{-1}$ in a potential range of $\pm 50 \mathrm{mV}$ around the open-circuit potential (OCP) of the sample in $1 \mathrm{M} \mathrm{HClO}_{4}$ vs. a $\mathrm{Ag} / \mathrm{AgCl}$ reference electrode (Figure S2-A). The capacitance (obtained from the averaged slope of the anodic and cathodic charging currents at OCP vs. scan rate) was scaled with the area-specific capacitance $\left(40 \mu \mathrm{F} \mathrm{cm}^{-2}\right)^{63,64}$ to obtain the ECSA (Figure S2B). The current in CV (Figure 3c) was normalized to the AuNP ECSA determined with $6.69 \mathrm{~cm}^{2}$ to give the current density $j$. For (photo-)electrochemical tests the cell (reference electrode was changed to a $\mathrm{Ag}$ wire due to the change in $\mathrm{pH}$ from acidic to alkaline) was first filled with freshly degassed $500 \mathrm{mM}$ glucose solution $+500 \mathrm{mM} \mathrm{KOH}$ aqueous solution. $\mathrm{CV}$ experiments were performed under dark conditions for three successive cycles at $50 \mathrm{mV} \mathrm{s}^{-1}$ scan rate. Subsequent current transients were recorded for $60 \mathrm{~s}$ under dark and illuminated conditions in the following order: 1) for varied potentials under $454 \mathrm{~nm}$ illumination ( $15.3 \mathrm{~mW}$, supplied by a tunable light source TLS03 by Zahner, Germany, illuminating the full sample area), 2) for varied intensities at an externally applied potential $E=$ $+300 \mathrm{mV}$ vs. Ag at the same wavelength and 3) for varied wavelengths and intensities according to the maximum available emission spectrum of the TLS03 (see Figure S5) at $E=+300 \mathrm{mV}$. After this procedure, the electrolyte was removed, the electrode cleaned thoroughly with water and steps 1) - 3) were repeated under freshly degassed $500 \mathrm{mM} \mathrm{KOH}$ solution. In addition, in repeated experiments with fresh substrates, no deviations of the experiment results were observed. In control experiments, the ITO substrate neither contributes to the observed dark nor to the photocurrent transients (see Supporting Information Figure S7) and was therefore regarded as passive.

\section{References}

(1) Halas, N. J. Spiers Memorial Lecture. Introductory lecture: Hot-electron science and microscopic processes in plasmonics and catalysis. Faraday Discuss. 2019, 214, 13-33.

(2) Graf, M.; Jalas, D.; Weissmüller, J.; Petrov, A. Y.; Eich, M. Surface-to-Volume Ratio Drives Photoelelectron Injection from Nanoscale Gold into Electrolyte. ACS Catal. 2019, 9, 3366-3374.

(3) Gellé, A.; Moores, A. Plasmonic nanoparticles: Photocatalysts with a bright future. Current Opinion in Green and Sustainable Chemistry 2019, 15, 60-66.

(4) Brongersma, M. L.; Halas, N. J.; Nordlander, P. Plasmon-induced hot carrier science and technology. Nat. Nanotechnol. 2015, 10, 25-34.

(5) Clavero, C. Plasmon-induced hot-electron generation at nanoparticle/metal-oxide interfaces for photovoltaic and photocatalytic devices. Nat. Photonics 2014, 8, 95-103.

(6) Wang, P.; Huang, B.; Dai, Y.; Whangbo, M.-H. Plasmonic photocatalysts: Harvesting visible light with noble metal nanoparticles. Phys. Chem. Chem. Phys. 2012, 14, 9813-9825.

(7) Christopher, P.; Xin, H.; Linic, S. Visible-light-enhanced catalytic oxidation reactions on plasmonic silver nanostructures. Nat. Chem. 2011, 3, 467-472.

(8) Christopher, P.; Xin, H.; Marimuthu, A.; Linic, S. Singular characteristics and unique chemical bond activation mechanisms of photocatalytic reactions on plasmonic nanostructures. Nat. Mater. 2012, 11, 1044-1050. 
(9) Hartland, G. V.; Besteiro, L. V.; Johns, P.; Govorov, A. O. What's so Hot about Electrons in Metal Nanoparticles? ACS Energy Lett. 2017, 2, 1641-1653.

(10) Brown, A. M.; Sundararaman, R.; Narang, P.; Schwartzberg, A. M.; Goddard, W. A.; Atwater, H. A. Experimental and Ab Initio Ultrafast Carrier Dynamics in Plasmonic Nanoparticles. Phys. Rev. Lett. 2017, 118, 87401.

(11) Brown, A. M.; Sundararaman, R.; Narang, P.; Goddard, W. A.; Atwater, H. A. Ab initio phonon coupling and optical response of hot electrons in plasmonic metals. Phys. Rev. B 2016, 94, 776.

(12) Khurgin, J. B. Hot carriers generated by plasmons: Where are they generated and where do they go from there? Faraday Discuss. 2019, 214, 35-58.

(13) Boriskina, S. V.; Cooper, T. A.; Zeng, L.; Ni, G.; Tong, J. K.; Tsurimaki, Y.; Huang, Y.; Meroueh, L.; Mahan, G.; Chen, G. Losses in plasmonics: From mitigating energy dissipation to embracing loss-enabled functionalities. Adv. Opt. Photon. 2017, 9, 775.

(14) Bernardi, M.; Mustafa, J.; Neaton, J. B.; Louie, S. G. Theory and computation of hot carriers generated by surface plasmon polaritons in noble metals. Nat. Commun. 2015, 6, 7044.

(15) Kelly, K. L.; Coronado, E.; Zhao, L. L.; Schatz, G. C. The Optical Properties of Metal Nanoparticles: The Influence of Size, Shape, and Dielectric Environment. J. Phys. Chem. B 2003, 107, 668-677.

(16) Seemala, B.; Therrien, A. J.; Lou, M.; Li, K.; Finzel, J. P.; Qi, J.; Nordlander, P.; Christopher, P. Plasmon-Mediated Catalytic O2 Dissociation on Ag Nanostructures: Hot Electrons or Near Fields? ACS Energy Lett. 2019, 4, 1803-1809.

(17) Dal Forno, S.; Ranno, L.; Lischner, J. Material, Size, and Environment Dependence of PlasmonInduced Hot Carriers in Metallic Nanoparticles. J. Phys. Chem. C 2018, 122, 8517-8527.

(18) Linic, S.; Aslam, U.; Boerigter, C.; Morabito, M. Photochemical transformations on plasmonic metal nanoparticles. Nat. Mater. 2015, 14, 567-576.

(19) Baffou, G.; Quidant, R. Thermo-plasmonics: Using metallic nanostructures as nano-sources of heat. Laser \& Photonics Rev. 2013, 7, 171-187.

(20) Hogan, N. J.; Urban, A. S.; Ayala-Orozco, C.; Pimpinelli, A.; Nordlander, P.; Halas, N. J. Nanoparticles heat through light localization. Nano Lett. 2014, 14, 4640-4645.

(21) Boerigter, C.; Aslam, U.; Linic, S. Mechanism of Charge Transfer from Plasmonic Nanostructures to Chemically Attached Materials. ACS Nano 2016, 10, 6108-6115.

(22) Pensa, E.; Gargiulo, J.; Lauri, A.; Schlücker, S.; Cortés, E.; Maier, S. A. Spectral Screening of the Energy of Hot Holes over a Particle Plasmon Resonance. Nano Lett. 2019, 19, 1867-1874.

(23) Wang, C.; Nie, X.-G.; Shi, Y.; Zhou, Y.; Xu, J.-J.; Xia, X.-H.; Chen, H.-Y. Direct PlasmonAccelerated Electrochemical Reaction on Gold Nanoparticles. ACS Nano 2017, 11, 5897-5905.

(24) Ortega-Liebana, M. C.; Hueso, J. L.; Arenal, R.; Santamaria, J. Titania-coated gold nanorods with expanded photocatalytic response. Enzyme-like glucose oxidation under near-infrared illumination. Nanoscale 2017, 9, 1787-1792.

(25) Schlather, A. E.; Manjavacas, A.; Lauchner, A.; Marangoni, V. S.; DeSantis, C. J.; Nordlander, P.; Halas, N. J. Hot Hole Photoelectrochemistry on Au@SiO2@Au Nanoparticles. J. Phys. Chem. Lett. 2017, 8, 2060-2067.

(26) Zhao, J.; Nguyen, S. C.; Ye, R.; Ye, B.; Weller, H.; Somorjai, G. A.; Alivisatos, A. P.; Toste, F. D. A Comparison of Photocatalytic Activities of Gold Nanoparticles Following Plasmonic and Interband Excitation and a Strategy for Harnessing Interband Hot Carriers for Solution Phase Photocatalysis. ACS Cent. Sci. 2017, 3, 482-488.

(27) Kale, M. J.; Avanesian, T.; Christopher, P. Direct Photocatalysis by Plasmonic Nanostructures. ACS Catal. 2013, 4, 116-128.

(28) Kim, Y.; Dumett Torres, D.; Jain, P. K. Activation Energies of Plasmonic Catalysts. Nano Lett. 2016, 16, 3399-3407.

(29) Baumberg, J. J. Hot electron science in plasmonics and catalysis: What we argue about. Faraday Discuss. 2019, 214, 501-511.

(30) Sivan, Y.; Un, I. W.; Dubi, Y. Assistance of metal nanoparticles in photocatalysis - nothing more than a classical heat source. Faraday Discuss. 2019, 214, 215-233. 
(31) Dubi, Y.; Sivan, Y. "Hot" electrons in metallic nanostructures-non-thermal carriers or heating? Light Sci. Appl. 2019, 8, 89.

(32) Zhang, X.; Li, X.; Reish, M. E.; Du Zhang; Su, N. Q.; Gutiérrez, Y.; Moreno, F.; Yang, W.; Everitt, H. O.; Liu, J. Plasmon-Enhanced Catalysis: Distinguishing Thermal and Nonthermal Effects. Nano Lett. 2018, 18, 1714-1723.

(33) Yu, Y.; Sundaresan, V.; Willets, K. A. Hot Carriers versus Thermal Effects: Resolving the Enhancement Mechanisms for Plasmon-Mediated Photoelectrochemical Reactions. J. Phys. Chem. C 2018, 122, 5040-5048.

(34) Zhou, L.; Swearer, D. F.; Zhang, C.; Robatjazi, H.; Zhao, H.; Henderson, L.; Dong, L.; Christopher, P.; Carter, E. A.; Nordlander, P. et al. Quantifying hot carrier and thermal contributions in plasmonic photocatalysis. Science 2018, 362, 69.

(35) Mukherjee, S.; Libisch, F.; Large, N.; Neumann, O.; Brown, L. V.; Cheng, J.; Lassiter, J. B.; Carter, E. A.; Nordlander, P.; Halas, N. J. Hot electrons do the impossible: Plasmon-induced dissociation of $\mathrm{H} 2$ on Au. Nano Lett. 2013, 13, 240-247.

(36) Zhan, C.; Liu, B.-W.; Huang, Y.-F.; Hu, S.; Ren, B.; Moskovits, M.; Tian, Z.-Q. Disentangling charge carrier from photothermal effects in plasmonic metal nanostructures. Nat. Commun. 2019, $10,2671$.

(37) Li, X.; Everitt, H. O.; Liu, J. Confirming nonthermal plasmonic effects enhance CO2 methanation on $\mathrm{Rh} / \mathrm{TiO} 2$ catalysts. Nano Res. 2019, 12, 1906-1911.

(38) Zuloaga, J.; Nordlander, P. On the energy shift between near-field and far-field peak intensities in localized plasmon systems. Nano Lett. 2011, 11, 1280-1283.

(39) Pinchuk, A.; Plessen, G. v.; Kreibig, U. Influence of interband electronic transitions on the optical absorption in metallic nanoparticles. J. Phys. D: Appl. Phys. 2004, 37, 3133-3139.

(40) Michaels, A. M.; Jiang; Brus, L. Ag Nanocrystal Junctions as the Site for Surface-Enhanced Raman Scattering of Single Rhodamine 6G Molecules. J. Phys. Chem. B 2000, 104, 1196511971.

(41) Viets, C.; Hill, W. Laser Power Effects in SERS Spectroscopy at Thin Metal Films. J. Phys. Chem. B 2001, 105, 6330-6336.

(42) Zheng, X.-S.; Hu, P.; Zhong, J.-H.; Zong, C.; Wang, X.; Liu, B.-J.; Ren, B. Laser Power Dependent Surface-Enhanced Raman Spectroscopic Study of 4-Mercaptopyridine on Uniform Gold Nanoparticle-Assembled Substrates. J. Phys. Chem. C 2014, 118, 3750-3757.

(43) Etchegoin, P. G.; Le Ru, E. C.; Meyer, M. An analytic model for the optical properties of gold. $J$. Chem. Phys. 2006, 125, 164705.

(44) Khurgin, J. B. How to deal with the loss in plasmonics and metamaterials. Nat. Nanotechnol. 2015, 10, 2-6.

(45) Brown, A. M.; Sundararaman, R.; Narang, P.; Goddard, W. A.; Atwater, H. A. Nonradiative Plasmon Decay and Hot Carrier Dynamics: Effects of Phonons, Surfaces, and Geometry. ACS Nano 2016, 10, 957-966.

(46) Minutella, E.; Schulz, F.; Lange, H. Excitation-Dependence of Plasmon-Induced Hot Electrons in Gold Nanoparticles. J. Phys. Chem. Lett. 2017, 8, 4925-4929.

(47) Graf, M.; Haensch, M.; Carstens, J.; Wittstock, G.; Weissmüller, J. Electrocatalytic methanol oxidation with nanoporous gold: Microstructure and selectivity. Nanoscale 2017, 9, 1783917848.

(48) Wang, J.; Cao, X.; Wang, X.; Yang, S.; Wang, R. Electrochemical Oxidation and Determination of Glucose in Alkaline Media Based on Au (111)-Like Nanoparticle Array on Indium Tin Oxide Electrode. Electrochim. Acta 2014, 138, 174-186.

(49) Karra, S.; Wooten, M.; Griffith, W.; Gorski, W. Morphology of Gold Nanoparticles and Electrocatalysis of Glucose Oxidation. Electrochim. Acta 2016, 218, 8-14.

(50) Tominaga, M.; Shimazoe, T.; Nagashima, M.; Taniguchi, I. Electrocatalytic oxidation of glucose at gold nanoparticle-modified carbon electrodes in alkaline and neutral solutions.

Electrochemistry Communications 2005, 7, 189-193. 
(51) Kwon, Y.; Lai, S. C. S.; Rodriguez, P.; Koper, M. T. M. Electrocatalytic oxidation of alcohols on gold in alkaline media: Base or gold catalysis? J. Am. Chem. Soc. 2011, 133, 6914-6917.

(52) Assiongbon, K. A.; Roy, D. Electro-oxidation of methanol on gold in alkaline media: Adsorption characteristics of reaction intermediates studied using time resolved electro-chemical impedance and surface plasmon resonance techniques. Surf. Sci. 2005, 594, 99-119.

(53) Doyle, R. L.; Lyons, M. E. G. The mechanism of oxygen evolution at superactivated gold electrodes in aqueous alkaline solution. J. Solid State Electrochem. 2014, 18, 3271-3286.

(54) Pasta, M.; Ruffo, R.; Falletta, E.; Mari, C. M.; Della Pina, C. Alkaline glucose oxidation on nanostructured gold electrodes. Gold Bull. 2010, 43, 57-64.

(55) Redmond, P. L.; Brus, L. E. "Hot Electron" Photo-Charging and Electrochemical Discharge Kinetics of Silver Nanocrystals. J. Phys. Chem. C 2007, 111, 14849-14854.

(56) Mukherjee, S.; Zhou, L.; Goodman, A. M.; Large, N.; Ayala-Orozco, C.; Zhang, Y.; Nordlander, P.; Halas, N. J. Hot-electron-induced dissociation of $\mathrm{H} 2$ on gold nanoparticles supported on $\mathrm{SiO} 2$. J. Am. Chem. Soc. 2014, 136, 64-67.

(57) Sil, D.; Gilroy, K. D.; Niaux, A.; Boulesbaa, A.; Neretina, S.; Borguet, E. Seeing is believing: Hot electron based gold nanoplasmonic optical hydrogen sensor. ACS Nano 2014, 8, 7755-7762.

(58) Mao, Z.; Vang, H.; Garcia, A.; Tohti, A.; Stokes, B. J.; Nguyen, S. C. Carrier Diffusion-The Main Contribution to Size-Dependent Photocatalytic Activity of Colloidal Gold Nanoparticles. ACS Catal. 2019, 9, 4211-4217.

(59) Schulz, F.; Homolka, T.; Bastús, N. G.; Puntes, V.; Weller, H.; Vossmeyer, T. Little Adjustments Significantly Improve the Turkevich Synthesis of Gold Nanoparticles. Langmuir 2014, 30, 10779-10784.

(60) Schulz, F.; Vossmeyer, T.; Bastús, N. G.; Weller, H. Effect of the Spacer Structure on the Stability of Gold Nanoparticles Functionalized with Monodentate Thiolated Poly(ethylene glycol) Ligands. Langmuir 2013, 29, 9897-9908.

(61) Schulz, F.; Dahl, G. T.; Besztejan, S.; Schroer, M. A.; Lehmkühler, F.; Grübel, G.; Vossmeyer, T.; Lange, H. Ligand Layer Engineering To Control Stability and Interfacial Properties of Nanoparticles. Langmuir 2016, 32, 7897-7907.

(62) Mueller, N. S.; Vieira, B. G. M.; Schulz, F.; Kusch, P.; Oddone, V.; Barros, E. B.; Lange, H.; Reich, S. Dark Interlayer Plasmons in Colloidal Gold Nanoparticle Bi- and Few-Layers. ACS Photonics 2018, 5, 3962-3969.

(63) Trasatti, S.; Petrii, O. A. Real surface area measurements in electrochemistry. Pure Appl. Chem. 1991, 63, 711-734.

(64) Germain, P. S.; Pell, W. G.; Conway, B. E. Evaluation and origins of the difference between double-layer capacitance behaviour at Au-metal and oxidized Au surfaces. Electrochim. Acta 2004, 49, 1775-1788. 
Graphical Abstract:

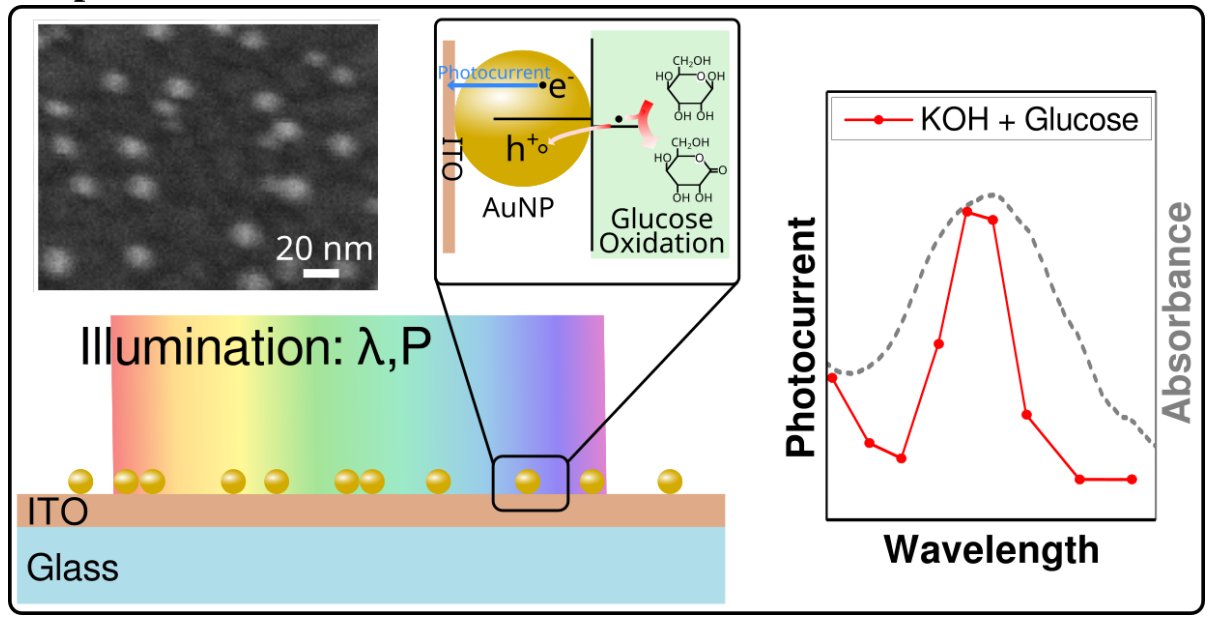

